\title{
Convex Games and Feasible Sets in Control Theory
}

\author{
Stefan Pickl \\ Department of Mathematics, University of Cologne \\ Weyertal 80, 50931 Cologne, email: pickl@zpr.uni-koeln.de \\ Dedicated to Werner Krabs at the occasion of his $66^{\text {th }}$ birthday
}

\begin{abstract}
The presented TEM-model describes the economical interaction between several actors (players) which intend to minimize their emissions $\left(E_{i}\right)$ caused by technologies $\left(T_{i}\right)$ by means of expenditures of money $\left(M_{i}\right)$ or financial means, respectively. The index stands for the $i$-th player, $i=1, \ldots, n$. The players are linked by technical cooperations and the market, which expresses itself in the nonlinear time-discrete dynamics of the Technology-Emissions-Means-model, in short: TEMmodel. In the sense of environmental protection, the aim is to reach a state which is mentioned in the Kyoto Protocol by choosing the control parameters such that the emissions of each player become minimized. The focal point is the realization of the necessary optimal control parameters via a played cost game, which is determined by the way of cooperation of the actors. In application to the work of Leitmann, but not regarding solution sets as feasible sets, the $\tau$-value of Tijs [13] is taken as a control parameter. This leads to a new class of problems in the area of 1-convex games. We want to solve the problem for a special case. With this solution a reasonable model for a Joint-Implementation process is developed, where its necessary fund is represented by the non-empty core of the analyzed game. Steering with parameters of this feasible set, the TEM-model can be regarded as a useful tool to implement and verify a technical Joint-Implementation Program. For the necessary data is given to the Clearing House, we are able to compare the numerical results with real world phenomena.
\end{abstract}

Keywords: Optimization, Convexity, Feasible Set, TEM-Model, $\tau$-value

\section{Introduction - The Kyoto Protocol}

The conferences of Rio de Janeiro 1992 and Kyoto 1997 demand for new economic instruments which have a focus on environmental protection in the macro and micro economy. An important economic tool being part of the Kyoto Protocol in that area is Joint-Implementation. It is an international program being part of the Kyoto Protocol which intends to strenghten international cooperations between enterprises in order to reduce $\mathrm{CO}_{2}$-reductions. A sustainable development can only be guaranteed if the instrument is embedded in an optimal energy management. Optimal energy management according to Joint-Implementation means in this context that it must work on a micro level with minimal costs and it should be protected against misuse on a macro level.

For that reason, the TEM-Model (Technology-Emissions-Means-Model) was developed [10], giving the possibility to simulate such an extraordinary market situation. The case of cooperative economic behavior including co-funding in joint international projects is as well considered as the mathematical analysis of several trend scenarios. This leads to new results in the area of cooperative time-discrete dynamic games using discrete optimization techniques and exploiting the underlying combinatorial structure. The realization of Joint-Implementation is determined by technical and financial constraints. In a Joint-Implementation Program the reduced emissions resulting from technical cooperations are registrated at the Clearing House. The associated cost reductions should then be allocated in an optimal way. 
This approach is as well integrated in the TEM-Model as the possibility to regard the influence of several cost-allocations on the feasible set of control parameters. In the played cost-game a special solution, namely the $\tau$-value $[13]$ which stands for a rational allocation process is examined. The main question is in which situations can the $\tau$-value be equivalent to the necessary control-parameters in order to reach the regions, which are mentioned in the Kyoto Protocol. Numerical results are as well shown as a qualitative analysis of the TEM-Model. The results in the area of cooperative dynamic games can lead to new insights in Joint-Implementation and can support an improvement of such an important economical management tool.

\section{The TEM-Model}

Technology-Emissions-Means-Model

The presented TEM-model describes the economical interaction between several actors (players) which intend to minimize their emissions $\left(E_{i}\right)$ caused by technologies $\left(T_{i}\right)$ by means of expenditures of money $\left(M_{i}\right)$ or financial means, respectively. The index stands for the $i$-th player, $i=1, \ldots, n$. The players are linked by technical cooperations and the market, which expresses itself in the nonlinear time-discrete dynamics of the Technology-Emissions-Means-model, in short: TEM-model.

We want to explain in short in which way can we describe the relationship between financial means and reduced emissions in a Joint-Implementation Program with the following equations:

$$
\begin{aligned}
E_{i}(t+1) & =E_{i}(t)+\sum_{j=1}^{n} e m_{i j}(t) M_{j}(t) \\
M_{i}(t+1) & =M_{i}(t)-\lambda_{i} M_{i}(t)\left[M_{i}^{*}-M_{i}(t)\right]\left\{E_{i}(t)+\varphi_{i} \Delta E_{i}(t)\right\} \\
E_{i} & \text { reduced emissions of actor i in percent } \\
M_{i} & \text { financial means of actor } \mathrm{i}
\end{aligned}
$$

Here, $e m_{i j}$ describes the effect on the emissions of the $i$-th actor, if the $j$-th actor invests money. We can say that it expresses how effective technology cooperations are, which is the central element of a Joint-Implementation Program. Furthermore, we are able to determine the $e m_{i j}$-parameter empirically. In the first equation the level of the reduced emissions at the $t$-th time-step depends upon the last value plus a market effect. This effect expresses itself in the additive terms which might be negative or positive. In general, $E_{i}>0$ implies that the actors have reached yet the demanded value $E_{i}=0$ (normalized Kyoto-Level). A value $E_{i}<0$ expresses that the emissions are less than the requirements of the treaty. In the second equation we see that for such a situation the financial means will increase whereas $E_{i}>0$ leads to a reduction of $M_{i}(t+1)$ :

$$
M_{i}(t+1)=M_{i}(t)-\lambda_{i} M_{i}(t)\left[M_{i}^{*}-M_{i}(t)\right]\left\{E_{i}(t)+\varphi_{i} \mathbf{\Delta} \mathbf{E}_{\mathbf{i}}(\mathbf{t})\right\}
$$

The equation contains the logistic functional dependence and the memory parameter $\varphi_{i}$ which describes the effect of the preceeding investment of financial means. The dynamics does not guarantee, that the parameter $M_{i}(t)$ lies in the interval, which can be regarded as a budget for the $i$-th actor

$$
0 \leq M_{i}(t) \leq M_{i}^{*}, \quad i=1, \ldots, n \quad \text { and } \quad t=0, \ldots, N
$$

For that reason we have to add restrictions to the dynamical representation. Then it is easy to show that

$$
-\lambda_{i} M_{i}(t)\left[M_{i}^{*}-M_{i}(t)\right] \leq 0 \quad \text { for } \quad i=1, \ldots, n \quad \text { and } \quad t=0, \ldots, N .
$$


We have guaranteed that $M_{i}(t+1)$ increases if $E_{i}(t)+\varphi_{i} \Delta E_{i}(t) \leq 0$ and decreases if $E_{i}(t)+\varphi_{i} \Delta E_{i}(t) \geq 0$. Applying the memory parameter $\varphi_{i}$ we have developed a reasonable model for the money expenditure - emission - interaction, where the influence of the technologies is integrated in the $e m$-matrix of the system. For an detailed description we might refer to [10] where the reader furthermore may found a detailed analysis of the TEM-model. The solutions of the following equations determine the fixed points of the TEM-model:

$$
\begin{aligned}
\sum_{j=1}^{n} e m_{i j} \hat{M}_{j}(t) & =0 \\
\hat{M}_{i}(t)\left[M_{i}^{*}-\hat{M}_{i}(t)\right] \hat{E}_{i}(t) & =0
\end{aligned}
$$

Regarding the Jakobi-matrix, we can state that the following eigenvalues

$$
\begin{gathered}
\mu_{1}=\ldots=\mu_{n}=1 \\
\mu_{n+j}=1-\lambda_{j} M_{j}^{*} \hat{E}_{j} \quad j=1, \ldots, n
\end{gathered}
$$

are not attractive.

\section{Numerical example}

In the following we will present two numerical examples which will give an intuition of the economical behavior which is given by the TEM-model. For the following data set

\begin{tabular}{|r|c|c|c|c|lcr|}
\hline & Emissions & Means & Budget & $\lambda$ & \multicolumn{3}{|c|}{$1 / 60 *$ em-Matrix } \\
\hline 1 & -0.1 & 30 & 60 & $1 / 60$ & 1 & -0.525 & -0.475 \\
2 & -0.1 & 20 & 60 & $1 / 60$ & -0.475 & 1 & 0.525 \\
3 & -0.1 & 10 & 60 & $1 / 60$ & -0.1 & -0.1 & 0.2 \\
\hline
\end{tabular}

we will observe the following oscillations of the reduced emissions and detect an increase of the financial means for player $1(-)$, player $2(-$.) and player $3(--)$.

emissions

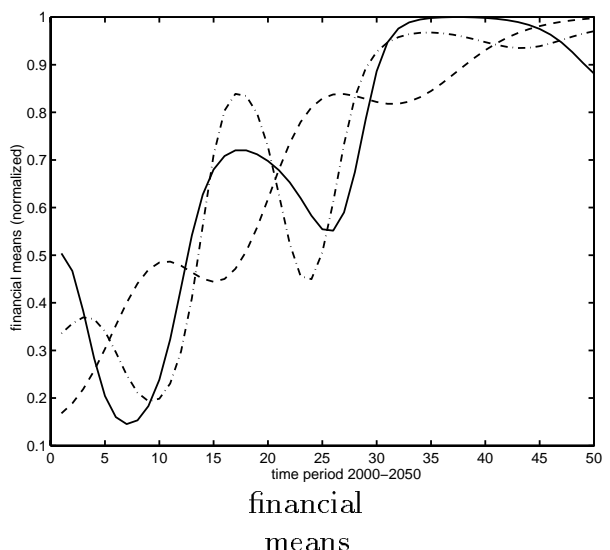

reduced

Additionally, for some data set, it is possible to observe chaotic behavior in the TEM-model. 


\begin{tabular}{|c|c|c|c|c|c|ccr|}
\hline Actor & Emissions & Means & Budget & $\varphi$ & $\lambda$ & \multicolumn{3}{|c|}{$e m$-Matrix } \\
\hline 1 & -1 & 0.3 & 1 & 11 & 0.82 & 1 & -0.7 & -0.3 \\
2 & 0.6 & 0.1 & 1 & 11 & 0.25 & -0.8 & 1 & -0.2 \\
3 & 0.5 & 0.2 & 1 & 11 & 0.4 & -0.9 & -0.1 & 1 \\
\hline
\end{tabular}
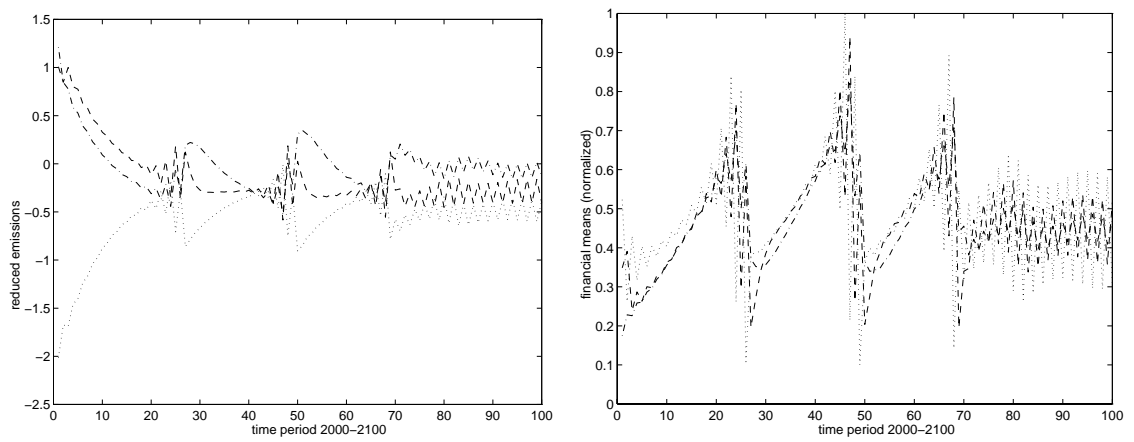

Even though this data is not relevant for practice, it demonstrates the necessity for a control theoretic approach.

\section{The Control Theory}

The numerical examinations underline the necessity of a control theoretic approach which is indicated by an additional control term in the second equation of the TEM-model.

$$
\begin{aligned}
E_{i}(t+1)= & E_{i}(t)+\sum_{j=1}^{n} e m_{i j} M_{j}(t) \\
M_{i}(t+1)= & M_{i}(t)-\lambda_{i} M_{i}(t)\left[M_{i}^{*}-M_{i}(t)\right] * \\
& {\left[E_{i}(t)+\varphi_{i} \Delta E_{i}(t)\right]+u_{i}(t) } \\
& u_{i}(t) \quad \text { control parameter }
\end{aligned}
$$

According to the Kyoto Protocol this approach means that each actor invests additional financial means. There are several possibilities to solve the problem of controllability. At this point, we want to concentrate on the feasible sets and their properties in the area of convex games. For that reason we present only one numerical result which shows that it is possible to steer the system into the fixed points. For a detailed description we might refer to [10].

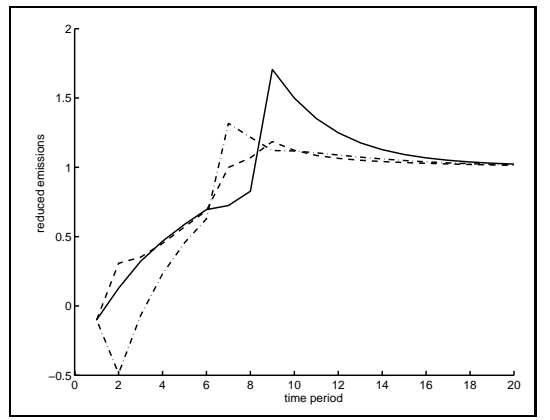




\section{The core as feasible set}

According to the introduction, optimal energy management in the sense of JointImplementation means that it must work on a micro level with minimal costs and it should be protected against misuse on a macro level. The first demand is solved by the control theoretic approach. The second subject can be treatened by adding constraints to the feasible set of our control parameters. A candidate might be the construction principle of the core in an cooperative n-person game which is defined in the following way:

\section{Definition 1}

Let $v$ be a n-person game with

$$
v: \operatorname{Pot}(\mathcal{N}) \rightarrow \mathbb{R}
$$

The Core $(v)$, is defined by

$$
\begin{aligned}
\operatorname{Core}(v):=\left\{y \in \mathbb{R}^{n} \mid \sum_{i \in \mathcal{N}} y_{i}=v(\mathcal{N})\right. & \text { und } \\
& \left.\sum_{i \in S} y_{i} \geq v(S) \quad(S \subseteq \mathcal{N})\right\}
\end{aligned}
$$

The core can be seen as a fund, which is constituted at each time-step by a cofunding process. This approach leads to the Bellmann Functional Equations, which are treatened in [10]. In the next section we will concentrate us on the construction of the underlying game. In application to the work of Leitmann [9], but not regarding solution sets as feasible sets, the $\tau$-value of Tijs [13], which has to lie in the core, is taken in the class of quasibalanced games, as a control parameter.

\section{The Cost-Game in the TEM-model}

If we regard the nonlinear time-discrete dynamics of the TEM-model

$$
\begin{aligned}
E_{i}(t+1)= & E_{i}(t)+\sum_{j=1}^{n} e m_{i j}(t) M_{j}(t) \\
M_{i}(t+1)= & M_{i}(t)-\lambda_{i} M_{i}(t)\left[M_{i}^{*}-M_{i}(t)\right]\left\{E_{i}(t)+\varphi_{i} \Delta E_{i}(t)\right\} \\
& \text { we can also formulate } \\
E_{i}(t+1)= & E_{i}(t)+\sum_{j=1}^{n} e m_{i j}(t) M_{j}(t) \\
M_{i}(t+1)= & M_{i}(t)-\lambda_{i} M_{i}(t)\left[M_{i}^{*}-M_{i}(t)\right]\left\{E_{i}(t)+\varphi_{i} \sum_{j=1}^{n} e m_{i j}(t) M_{j}(t)\right\} \\
& \text { considering that } \Delta E_{i}(t)=E_{i}(t+1)-E_{i}(t)
\end{aligned}
$$

In order to reach steady states, which are determined in [10], an independent institution may influence the trade relations between the actors.

In practice, the imposing of taxes or the giving of incentives means that in the TEMmodel the em-parameter will change. Now, the principle of Joint-Implementation implies that technical cooperation will be benefitted:

$$
\left(\begin{array}{ccc}
e m_{11} & e m_{12}+\epsilon & e m_{13} \\
e m_{21}+\epsilon & e m_{22} & e m_{23} \\
e m_{31} & e m_{32} & e m_{33}
\end{array}\right)
$$

Actor 1 and Actor 2 do cooperate

$$
\left(\begin{array}{ccc}
e m_{11}+\omega & e m_{12}+\omega & e m_{13}+\omega \\
e m_{21}+\omega & e m_{22}+\omega & e m_{23}+\omega \\
e m_{31}+\omega & e m_{32}+\omega & e m_{33}+\omega
\end{array}\right)
$$

All players do cooperate 
For $K_{i}(t) \lambda_{i} M_{i}(t) \geq 0$ the difference between the cooperative and the non-cooperative case is always positive. So we have constructed a reasonable cost-game. Now, the method is that at each time step, this amount is put into a central fund. We determine the core for this set and with the $\tau$-value, which has to lie in it, we want to steer the system. Such a determined value can be considered as a favourite candidate of a co-funding process at each phase of a Joint-Implementation Program. Evaluation of determinants of environmental protection according to Joint-Implementation should be oriented on that approach. In the next section, we will give a short introduction into the theory of quasibalanced games which guarantees at least that the $\tau$-value is an element of the core.

\section{Quasibalanced Games}

For the convenience of the reader we repeat the relevant material from [3] and [14] without proofs, thus makes our exposition self contained. We assume that the reader is familiar with the terminology of the gap-function, the upper-vector and the concession vector which are introduced in [13] and [3].

Definition 2 The class $Q B^{n}$ of quasibalanced n-person games is given by

$$
\begin{gathered}
Q B^{n}:=\left\{v \in G^{n} \mid \lambda^{v}(\mathcal{N}) \geq g^{v}(\mathcal{N}) \quad \text { and } g^{v}(S) \geq 0 \quad \text { for all } S \in \operatorname{Pot}(\mathcal{N})\right\} \\
=\left\{v \in G^{n} \mid \begin{array}{l}
\lambda_{i}^{v} \geq 0 \quad \text { for all } \quad i \in \mathcal{N} \quad \text { and } \\
\left.\left(b^{v}-\lambda^{v}\right)(\mathcal{N}) \leq v(\mathcal{N}) \leq b^{v}(\mathcal{N})\right\}
\end{array}\right.
\end{gathered}
$$

Using the assumptions that we regard quasibalanced games, we can proof the following theorem

Theorem 1 Let $v \in G^{n}$ and the gap function has the following property $g^{v}(S) \geq 0$ for all $S \in \operatorname{Pot}(\mathcal{N})$. Furthermore we have $g^{v}(\mathcal{N})=0$. Then $\tau \in \operatorname{Core}(v)$.

The main idea of the proof is to take the $\tau$-value as a core element. The detailed proof is left to the reader.

Using the $\tau$-value as a control parameter we have to present necessary and sufficient conditions for the $\tau$-value to belong to the core. In the following we will concentrate upon quasi-balanced games and regard the core as feasible set of our control problem.

Theorem 2 Let $v \in Q B^{v}$. If $g^{v}(\mathcal{N})=0$ then $\tau \in$ Core $(v)$.

Furthermore Core $(v)=\{\tau(v)\}$.

Theorem 3 Let $v \in Q B^{n}$ and $\lambda^{v}(S)>0, S \in \operatorname{Pot}(\mathcal{N})$. The $\tau$-value is element of the Core iff the following criteria is valid:

$$
\frac{g^{v}(S)}{\lambda^{v}(S)} \geq \frac{g^{v}(\mathcal{N})}{\lambda^{v}(\mathcal{N})}, S \in \operatorname{Pot}(\mathcal{N})
$$

Furthermore it is possible to prove the theorem under the assumption that the gap function is positive, i.e. $g^{v}(S)>0$. This condition implies that there exists in each set $S \in \operatorname{Pot}(\mathcal{N})$ an element $i^{*}$ such that the corresponding concession vector $\lambda_{i^{*}}^{v}$ is positive. For this is valid for all sets $S \in \operatorname{Pot}(\mathcal{N})$ we can conclude that

$$
\forall_{i \in \mathcal{N}}: \quad \lambda_{i}^{v}>0 \quad \text { which implies that } \quad \forall S \in P \text { ot }(\mathcal{N}): \quad \lambda^{v}(S)>0 .
$$

Lemma 1 Let $v \in G^{n}$. Then $g^{v}(\mathcal{N})=g^{v}(\mathcal{N} \backslash\{i\})$ for all $i \in \mathcal{N}$. 
Theorem 4 Let $v \in Q B^{n}, g^{v}(\mathcal{N})>0$ and the condition of Theorem 3

$$
\frac{g^{v}(S)}{\lambda^{v}(S)} \geq \frac{g^{v}(\mathcal{N})}{\lambda^{v}(\mathcal{N})}
$$

which gives a sufficient and necessity condition that the $\tau$-value is an element of the core. In order to check (7.1) and (7.2) it is sufficient to examine only the coalitions $2 \leq|S| \leq n-1, S \in \operatorname{Pot}(\mathcal{N})$.

Theorem 5 Let $v \in Q B^{n}$ and the number of players $n \in\{1,2,3\}$. Under these assumptions the $\tau$-value is element of the Core, i.e.

$$
\tau \in \operatorname{Core}(v) \text {. }
$$

Definition 3 The class of 1-convex games is characterized by the fact that all actors have the same concession vector $\lambda_{i}^{v}$ with $\lambda_{i}^{v} \geq 0$ :

$$
\lambda_{i}^{v}=g^{v}(\mathcal{N}), i=1, \ldots, n
$$

By that definition, the concession vector is characterized merely by the grand coalition, i.e. the gap of the grand coalition $\mathcal{N}$ is minimal among the gaps of nonempty coalitions.

Theorem 6 Let $v \in C_{1}^{n}$ and $1_{n}=(1,1, \ldots, 1)^{T} \in \mathbb{R}^{n}$. Then $v \in Q B^{n}$ and

$$
\tau(v)=b^{v}-\frac{g^{v}(\mathcal{N}) 1_{n}}{n} \in \operatorname{Core}(v) .
$$

The results of Tijs and Driessen [13] and [3] of this section have nice properties and their clearness tends to a direct application in the TEM-Model. Nevertheless, our next goal is to sharphen these results in the context of the TEM-Model and the underlying cost-game. The aim is to get a representation for our set of the control parameters which can then be realized by a played cost-game whereas the feasible set is presented by the core.

\section{The Cost-game}

According to ( 6.1 ) and (6.2) let us begin with the construction of the cost-game in the TEM-model

$$
\begin{aligned}
v_{t}(K) & :=\underbrace{\sum_{j \in K} M_{j}(t)}_{\text {Without Cooperation }}-\underbrace{M(K)}_{\text {Cooperation }} \\
& =\left(\begin{array}{lll}
K_{1}^{*}(t) & K_{2}^{*}(t) & K_{3}^{*}(t)
\end{array}\right)\left(\begin{array}{ccc}
0 & \epsilon & \delta \\
\epsilon & 0 & \gamma \\
\delta & \gamma & 0
\end{array}\right)_{\operatorname{Ind}(K)}\left(\begin{array}{l}
M_{1}(t) \\
M_{2}(t) \\
M_{3}(t)
\end{array}\right)
\end{aligned}
$$

with $K_{i}^{*}(t)=\varphi_{i} \tilde{M}_{i}(t)(i=1, \ldots, n)$ and $K \in \operatorname{Pot}(\mathcal{N})$. In the sequel, we have

$$
(B)_{\operatorname{Ind}(K)}:=A, \text { with }\left\{\begin{array}{l}
a_{i j}=b_{i j}, \text { if } \quad i \in K \quad \text { and } \quad j \in K \\
a_{i j}=0
\end{array}\right.
$$

For the grand coalition we get:

$$
\begin{aligned}
v_{t}(\mathcal{N}) & :=\underbrace{\sum_{j \in \mathcal{N}} M_{j}(t)}_{\text {Without Cooperation }}-\underbrace{M(\mathcal{N})}_{\text {Cooperation }} \\
& =\left(\begin{array}{lll}
K_{1}^{*}(t) & K_{2}^{*}(t) & K_{3}^{*}(t)
\end{array}\right)\left(\begin{array}{ccc}
0 & \omega & \omega \\
\omega & 0 & \omega \\
\omega & \omega & 0
\end{array}\right)\left(\begin{array}{l}
M_{1}(t) \\
M_{2}(t) \\
M_{3}(t)
\end{array}\right)
\end{aligned}
$$


For simplicity of notation, we write:

$$
\begin{aligned}
\tilde{v}(\omega) & :=v(\{1,2,3\})(\omega) \\
\tilde{v}(\epsilon) & :=v(\{1,2\})(\epsilon) \\
\tilde{v}(\delta) & :=v(\{1,3\})(\delta) \\
\tilde{v}(\gamma) & :=v(\{2,3\})(\gamma)
\end{aligned}
$$

Furthermore we will neglect the index $t$. For the game will be played at each timestep separately, we can procede in that way without loss restriction of generality. We will assume that our game is super-additive. In the following we want to examine for which parameters our game is 1-convex.

Theorem 7 Let $\tilde{v}$ be the game defined by (8.1). The game $\tilde{v}$ is 1-convex, if

$$
\begin{aligned}
\tilde{v}(\omega) & =\max \{\tilde{v}(\epsilon), \tilde{v}(\delta), \tilde{v}(\gamma)\} \\
& =\min \{\tilde{v}(\epsilon)+\tilde{v}(\delta), \tilde{v}(\epsilon)+\tilde{v}(\gamma), \tilde{v}(\gamma)+\tilde{v}(\delta)\} .
\end{aligned}
$$

Proof 7 First of all, we have to prove that $\tilde{v}(\mathcal{N})-b^{\tilde{v}}(\mathcal{N} \backslash S) \geq \tilde{v}(S)$, for all $S \in$ $\operatorname{Pot}(\mathcal{N})$.

Let us begin with $S=\{1,2,3\}$. Then we get

$$
\tilde{v}(\{1,2,3\})-b^{\tilde{v}}(\varnothing)=\tilde{v}(\{1,2,3\}),
$$

which is precisely the assertion.

Now we will turn to the coalition $S=\{1,2\}$. We get $\tilde{v}(\{1,2,3\})-b^{\tilde{v}}(\{3\})=$ $\tilde{v}(\{1,2,3\})-[\tilde{v}(\{1,2,3\})-\tilde{v}(\{1,2\})]$, which is again the assertion.

It lasts to show wether $\tilde{v}(\{1,2,3\})-b^{\tilde{v}}(\{2,3\}) \geq v(\{i\})(i=1, \ldots, n)$. For the game is zero-normalized we receive for the coalition $S=\{1\}$ the condition $\tilde{v}(\{1,2,3\})-$ $b^{\tilde{v}}(\{2,3\}) \geq 0$ and equivalently $\tilde{v}(\{1,2,3\})-[\tilde{v}(\{1,2,3\})-\tilde{v}(\{1,3\})+\tilde{v}(\{1,2,3\})-$ $\tilde{v}(\{1,2\})] \geq 0$. With

$$
\tilde{v}(\{1,2,3\})=\min \{\tilde{v}(\epsilon)+\tilde{v}(\delta), \tilde{v}(\epsilon)+\tilde{v}(\gamma), \tilde{v}(\gamma)+\tilde{v}(\delta)\}
$$

we obtain a sufficient condition.

Secondly, we have to show wether the condition $b^{\tilde{v}}(\mathcal{N}) \geq \tilde{v}(\mathcal{N})$ is valid for 1 convexity. By the definition we get

$$
\tilde{v}(\omega) \geq \frac{\tilde{v}(\epsilon)+\tilde{v}(\delta)+\tilde{v}(\gamma)}{2}
$$

Without loss restriction of generality we can assume that the minimum of

$$
\min \{\tilde{v}(\epsilon)+\tilde{v}(\delta), \tilde{v}(\epsilon)+\tilde{v}(\gamma), \tilde{v}(\gamma)+\tilde{v}(\delta)\}
$$

is attained by $\tilde{v}(\epsilon)+\tilde{v}(\delta)$. Furthermore it lasts $\tilde{v}(\gamma) \leq \max \{\tilde{v}(\epsilon), \tilde{v}(\delta), \tilde{v}(\gamma)\}$. This finishes the proof.

Now we are able to compute the $\tau$-value in this 1-convex game.

$$
\begin{aligned}
\tau & =b^{\tilde{v}}(\mathcal{N})-\frac{1}{n} g(\mathcal{N}) \\
& =\left(\begin{array}{l}
\tilde{v}(\omega)-\tilde{v}(\gamma)-\frac{1}{3} g^{\tilde{v}}(\omega) \\
\tilde{v}(\omega)-\tilde{v}(\delta)-\frac{1}{3} g^{\tilde{v}}(\omega) \\
\tilde{v}(\omega)-\tilde{v}(\epsilon)-\frac{1}{3} g^{\tilde{v}}(\omega)
\end{array}\right) \\
& =\left(\begin{array}{l}
\frac{1}{3} \tilde{v}(\omega)+\frac{1}{3} \tilde{v}(\epsilon)+\frac{1}{3} \tilde{v}(\delta)-\frac{2}{3} \tilde{v}(\gamma) \\
\frac{1}{3} \tilde{v}(\omega)+\frac{1}{3} \tilde{v}(\epsilon)+\frac{1}{3} \tilde{v}(\gamma)-\frac{2}{3} \tilde{v}(\delta) \\
\frac{1}{3} \tilde{v}(\omega)+\frac{1}{3} \tilde{v}(\delta)+\frac{1}{3} \tilde{v}(\gamma)-\frac{2}{3} \tilde{v}(\epsilon)
\end{array}\right) !\left(\begin{array}{l}
u_{1} \\
u_{2} \\
u_{3}
\end{array}\right)
\end{aligned}
$$


Our next concern is to find a solution of the following system of equations:

$$
\left(\begin{array}{l}
\max \{x, y, z\} \\
\max \{x, y, z\} \\
\max \{x, y, z\}
\end{array}\right)+\left(\begin{array}{ccc}
1 & 1 & -2 \\
1 & -2 & 1 \\
-2 & 1 & 1
\end{array}\right)\left(\begin{array}{l}
x \\
y \\
z
\end{array}\right)=\left(\begin{array}{l}
3 u_{1} \\
3 u_{2} \\
3 u_{3}
\end{array}\right)
$$

The solution set can be seen as the feasible set of our control problem where each feasible control vector has to be equivalent to the $\tau$-value of a played convex game. In the following we want to characterize this set.

\section{A characterization of the feasible set}

Theorem 8 Let $v$ be the cost game which is defined in (8.1). Assuming that we distinguish the following cases of the nondifferentiable system (8.4) of equations. We get then the following solutions.

1. Assumption: Let the maximum be attained at $x$

$$
\begin{gathered}
x=u_{1}+u_{2}+u_{3} \quad y=u_{1}+2 u_{3} \quad z=2 u_{3}+u_{2} \\
\text { with } \quad u_{1} \geq u_{3}(x \geq z) \quad \text { and } \quad u_{2} \geq u_{3}(x \geq y)
\end{gathered}
$$

2. Assumption: Let the maximum be attained at $y$

$$
\begin{gathered}
x=2 u_{2}+u_{1} \quad y=u_{1}+u_{2}+u_{3} \quad z=2 u_{2}+u_{3} \\
\text { with } \quad u_{1} \geq u_{2}(y \geq z) \quad \text { and } \quad u_{3} \geq u_{2}(y \geq x)
\end{gathered}
$$

3. Assumption: Let the maximum be attained at $z$

$$
\begin{gathered}
x=2 u_{1}+u_{2} \quad y=2 u_{1}+u_{3} \quad z=u_{1}+u_{2}+u_{3} \\
\text { with } \quad u_{2} \geq u_{1}(z \geq y) \quad \text { and } \quad u_{3} \geq u_{1}(z \geq x)
\end{gathered}
$$

If the control parameter have the following properties

1. $u_{1} \geq 0 \quad u_{2} \geq 0 \quad u_{3}=0$

2. $u_{1} \geq 0 \quad u_{2}=0 \quad u_{3} \geq 0$

3. $u_{1}=0 \quad u_{2} \geq 0 \quad u_{3} \geq 0$

then we can construct for each case an equivalent 1-convex game.

Proof 8 First of all we have to solve the system of equations for each case. Let us make the following distinctions. Under the conditions stated above, we get

1. Assumption: Let the maximum be attained at $x$ Then we have $x=u_{1}+u_{2}+u_{3} \quad y=u_{1}+2 u_{3} \quad z=2 u_{3}+u_{2}$.

2. Assumption: Let the maximum be attained at $y$ Then we have $x=2 u_{2}+u_{1} \quad y=u_{1}+u_{2}+u_{3} \quad z=2 u_{2}+u_{3}$.

3. Assumption: Let the maximum be attained at $z$

Then we have $x=2 u_{1}+u_{2} \quad y=2 u_{1}+u_{3} \quad z=u_{1}+u_{2}+u_{3}$.

Under the hypotheses, mentioned above, we have to prove, wether the conditions are valid. 
Let us begin with the first case.

- First case: Let the maximum be attained at $x$ we get $x \geq z:$

$$
\begin{aligned}
u_{1}+u_{2}+u_{3} & \geq 2 u_{3}+u_{2} \\
u_{1} & \geq u_{3}
\end{aligned}
$$

$x \geq y:$

$$
\begin{aligned}
u_{1}+u_{2}+u_{3} & \geq 2 u_{3}+u_{1} \\
u_{2} & \geq u_{3}
\end{aligned}
$$

This leads to the constraints $u_{1} \geq u_{3}$ und $u_{2} \geq u_{3}$.

- Second case: Let the maximum be attained at $y$ we get

$y \geq x:$

$$
\begin{aligned}
u_{1}+u_{2}+u_{3} & \geq 2 u_{2}+u_{1} \\
u_{3} & \geq u_{2}
\end{aligned}
$$

$y \geq z:$

$$
\begin{aligned}
u_{1}+u_{2}+u_{3} & \geq u_{3}+2 u_{2} \\
u_{1} & \geq u_{2}
\end{aligned}
$$

This leads to the constraints $u_{3} \geq u_{2}$ und $u_{1} \geq u_{2}$.

- Third case: Let the maximum be attained at $z$ we get

$z \geq x$ :

$$
\begin{aligned}
u_{1}+u_{2}+u_{3} & \geq 2 u_{1}+u_{2} \\
u_{3} & \geq u_{1}
\end{aligned}
$$

$z \geq y:$

$$
\begin{aligned}
u_{1}+u_{2}+u_{3} & \geq 2 u_{1}+u_{3} \\
u_{2} & \geq u_{1}
\end{aligned}
$$

This leads to the constraints $u_{3} \geq u_{1}$ und $u_{2} \geq u_{1}$.

According to Theorem 7 it is sufficient for 1-convexity, that

$$
\max \{x, y, z\}=\min \{x+y, y+z, y+z\} .
$$

In the following we want to present the proof only for one of the three case-studies. The approach is similar in all the three cases. The other cases are left to the reader. Let us concentrate on the first case. According to that hypothese we get the constraints

$$
u_{1} \geq u_{3} \quad \text { and } \quad u_{2} \geq u_{3} .
$$

In addition to (9.1) and (9.2) we demand now that

$$
x=\min \{x+y, y+z, z+x\}
$$

Without loss restriction of generality, let us assume that the minimum is attained at $y+z$. Then we get

$$
\begin{aligned}
x & =y+z \\
u_{1}+u_{2}+u_{3} & =u_{1}+2 u_{3}+2 u_{3}+u_{2} \\
\Rightarrow & u_{3}=0
\end{aligned}
$$


Applying (9.3) we obtain $u_{1} \geq 0$ and $u_{2} \geq 0$.

From the above it follows that

$$
\begin{aligned}
& \min \{x+y, y+z, z+x\} \\
& =\min \left\{u_{1}+u_{2}+u_{3}+u_{1}+2 u_{3}, u_{1}+2 u_{3}+2 u_{3}+u_{2}, u_{1}+u_{2}+u_{3}+2 u_{3}+u_{2}\right\} \\
& =\min \left\{2 u_{1}+u_{2}, u_{1}+u_{2}, u_{1}+2 u_{2}\right\} \\
& =u_{1}+u_{2}=y+z
\end{aligned}
$$

In this way we see that for the case $u_{3}=0$ the minimum in (9.4) is attained at $y+z$. This completes the proof. The other cases are left to the reader.

\section{A special case - a three person game}

Now we will restrict our attention to the case where we have only three actors. According to the simulation of a Joint-Implementation Program, it is sufficient to deal with such a constellation. We are able to regard the interaction between the United States of America, the People's Republic of China and an European country. According to the above remarks, it was sufficient for the $\tau$-value being part of the core, restricted to three person games, that the game is quasibalanced, that means

$$
\lambda^{v}(\mathcal{N}) \geq g^{v}(\mathcal{N}) \quad \text { and } \quad g^{v}(S) \geq 0 .
$$

In comparison to the class of 1-convex games, it is not necessary that the minimum is attained by $g^{v}(\mathcal{N})$. For this reason, we can continue in the following way.

Let us start with the condition $g^{v}(S) \geq 0$. According to lemma 1 it is sufficient to consider the cases $g^{v}(\{i\}) \geq 0, i \in S$ and $g^{v}(\mathcal{N})$. Given any $i^{*} \in S$ then we can formulate

$$
g^{v}\left(\left\{i^{*}\right\}\right)=b_{i^{*}}^{v}-v\left(\left\{i^{*}\right\}\right)=v(\mathcal{N})-v\left(\mathcal{N} \backslash\left\{i^{*}\right\}\right)
$$

We will again assume that our system is super-additive. Additionally we will make the assumption that the cost-game is zero-normalized. In [10] the reader may find criteria which guarantee super-additivity and zero-normality. Basing on this presuppositions it follows that $g^{v}\left(\left\{i^{*}\right\}\right) \geq 0$.

Concerning the grand coalition $\{\mathcal{N}\}$, we may formulate:

$$
\begin{aligned}
g^{v}(\mathcal{N})= & b^{v}(\mathcal{N})-v(\mathcal{N}), \quad \mathcal{N}=\{1,2,3\} \\
= & b_{1}^{v}+b_{2}^{v}+b_{3}^{v}-v(\{1,2,3\}) \\
= & v(\mathcal{N})-v(\mathcal{N} \backslash\{2,3\})+v(\mathcal{N})-v(\mathcal{N} \backslash\{1,3\})+v(\mathcal{N})-v(\mathcal{N} \backslash\{1,2\}) \\
& -v(\{1,2,3\})
\end{aligned}
$$

We conclude from $v(\{1,2,3\}) \geq \frac{v(\{1,2\})+v(\{1,3\})+v(\{2,3\})}{2}$ that $g^{v}(\mathcal{N}) \geq 0$.

Applying the second condition for Quasibalancedness

$$
\begin{aligned}
\lambda(\mathcal{N}) & \geq g^{v}(\mathcal{N}) \\
\lambda_{1}^{v}+\lambda_{2}^{v}+\lambda_{3}^{v} & \geq g^{v}(\{1,2,3\}) \\
& \geq b_{1}^{v}+b_{2}^{v}+b_{3}^{v}-v(\{1,2,3\})
\end{aligned}
$$

leads to the following distinctions:

1. Let us begin with the case where the concession vector is, without loss restriction of generality, defined by $\lambda_{1}^{v}=g^{v}(\{1,2,3\})$.

For $g^{v}(S) \geq 0, S \in \mathcal{N}$ it yields $(10.1)$

2. We now turn to the case where the concession vector is, without loss restriction of generality, defined by $\lambda_{1}^{v}=g^{v}(\{1,2\})$.

According to lemma 1 we have $g^{v}(\{1,2\})=g^{v}(\{1,2,3\})$. From what has already been proved it follows that $(10.1)$ is valid. 
3. It remains to consider the case $\lambda_{1}^{v}=g^{v}(\{1\}), \lambda_{2}^{v}=g^{v}(\{2\}), \lambda_{3}^{v}=g^{v}(\{3\})$.

It is easily seen that

$$
\begin{array}{r}
b_{1}^{v}+b_{2}^{v}+b_{3}^{v}-v(\{1\})-v(\{2\})-v(\{3\})= \\
b_{1}^{v}+b_{2}^{v}+b_{3}^{v} \geq b^{v}(\{1,2,3\})-v(\{1,2,3\})=g^{v}(\mathcal{N})
\end{array}
$$

From what has already been proved we conclude that the condition

$$
v(\{1,2,3\}) \geq \frac{v(\{1,2\})+v(\{1,3\})+v(\{2,3\})}{2}
$$

represents again an equivalent problem. The crucial fact is that now only the condition ( 10.3 ) is valid. Under these conditions we may determine the $\tau$-value for an 3 -person quasibalanced game by:

$$
\begin{aligned}
& \tau_{1}=b_{1}^{v}-\frac{\lambda_{1}^{v}}{\lambda^{v}(\{1,2,3\})} g^{v}(\{1,2,3\}) \\
& \tau_{2}=b_{2}^{v}-\frac{\lambda_{2}^{v}}{\lambda^{v}(\{1,2,3\})} g^{v}(\{1,2,3\}) \\
& \tau_{3}=b_{3}^{v}-\frac{\lambda_{3}^{v}}{\lambda^{v}(\{1,2,3\})} g^{v}(\{1,2,3\})
\end{aligned}
$$

For the game is supposed to be zero-normalized, and we have

$$
\begin{aligned}
g^{v}(\{i\})= & v(\{1,2,3\})-v(\{1,2,3\} \backslash\{i\}) \\
& \text { and } \\
g^{v}(\{1,2,3\})= & b_{1}^{v}+b_{2}^{v}+b_{3}^{v}-v(\{1,2,3\}) \\
= & 2 v(\{1,2,3\})-v(\{1,2\})-v(\{1,3\})-v(\{2,3\}) \\
& \text { according to lemma } 1 \\
\lambda_{i}^{v}= & \min \left[g^{v}(\{i\}), g^{v}(\{1,2,3\})\right]
\end{aligned}
$$

For simplicity, let us introduce the following abbreviations,

$$
\begin{aligned}
& \lambda_{1}^{v}=\min \left\{g^{v}(\{1\}), g^{v}(\{1,2,3\})\right\}=f(x, y, z, w) \\
& \lambda_{2}^{v}= \min \left\{g^{v}(\{2\}), g^{v}(\{1,2,3\})\right\}=g(x, y, z, w) \\
& \lambda_{3}^{v}= \min \left\{g^{v}(\{3\}), g^{v}(\{1,2,3\})\right\}=h(x, y, z, w) \\
& \text { with } \quad x:=v(\{1,2\}) \quad y:=v(\{1,3\}) \quad z=v(\{2,3\}) \quad w=v(\{1,2,3\})
\end{aligned}
$$

According to this, we obtain the following representation

$$
\begin{aligned}
& \tau_{1}=w-z-\frac{f(x, y, z, w)}{f(x, y, z, w)+g(x, y, z, w)+h(x, y, z, w)}[2 w-x-y-z]:=u_{1} \\
& \tau_{2}=w-y-\frac{g(x, y, z, w)}{f(x, y, z, w)+g(x, y, z, w)+h(x, y, z, w)}[2 w-x-y-z]:=u_{2} \\
& \tau_{3}=w-x-\frac{h(x, y, z, w)}{f(x, y, z, w)+g(x, y, z, w)+h(x, y, z, w)}[2 w-x-y-z]:=u_{3}
\end{aligned}
$$

From (10.3) we obtain additionally as fourth condition for our so called equivalent problem:

$$
2 w-x-y-z=\epsilon, \epsilon \geq 0
$$


The equivalence set is the solution set of the following system of equations.

$$
\left(\begin{array}{c}
u_{1} \\
u_{2} \\
u_{3} \\
\epsilon
\end{array}\right)=\left(\begin{array}{cccc}
1-\frac{2 f}{f+g+h} & \frac{f}{f+g+h} & \frac{f}{f+g+h} & \frac{f}{f+g+h}-1 \\
1-\frac{2 g}{f+g+h} & \frac{g}{f+g+h} & \frac{g}{f+g+h}-1 & \frac{g}{f+g+h} \\
1-\frac{2 h}{f+g+h} & \frac{h}{f+g+h}-1 & \frac{h}{f+g+h} & \frac{h}{f+g+h}
\end{array}\right)\left(\begin{array}{c}
w \\
x \\
y \\
z
\end{array}\right.
$$

Numerical examples show that the solution set is not empty. This fact means that after a successive co-funding process which might be coordinated by the institution of the clearing house mentioned in the Kyoto Protocol it is possible to steer the control problem in an optimal way with the $\tau$-value. Additionaly the $\tau$-value lies in the core of an equivalent game. This is a new result in the area of dynamic cooperative games.

\section{References}

1. F.H. Clarke (1983) Optimization and Nonsmooth Analysis. New-York, Wiley Interscience

2. T.S.H. Driessen (1984) Game theoretic solutions for some economic situations. Cahiers du C.E.R.O., 26: 51-58

3. T.S.H. Driessen (1987) The $\tau$-value: a Survey. Surveys in game theory and related topics. Technical Report 39, CWI Tract: 181-208

4. H. Dyckhoff (2000) Umweltmanagement. Springer, Berlin

5. U. Faigle and W. Kern (1993) On some approximately balanced combinatorial cooperative games.

ZOR - Methods and Models of Operations Research 38: 141-152

6. G. Feichtinger and R.F. Hartl (1986) Optimale Kontrolle ökonomischer Prozesse. Anwendung des Maximumprinzips in den Wirtschaftswissenschaften. de Gruyter Verlag, Berlin

7. R. Hartl and P. Kort (1996) Capital accumulation of a firm facing on emissions tax. Journal of Economics, Vol. 63: 1-23

8. W. Krabs (1997) Mathematische Modellierung. B.G. Teubner, Stuttgart

9. G. Leitmann (1974) Cooperative and Non-Cooperative Many Players Differential Games. Springer Verlag, Wien, New York

10. S. Pickl (1999) Der $\tau$-value als Kontrollparameter. Modellierung und Analyse eines Joint-Implementation Programmes mithilfe der kooperativen dynamischen

Spieltheorie und der diskreten Optimierung. Shaker Verlag, Aachen

11. S. Pickl (2000) Controllability of the Time-Discrete TEM-model via the Solution of an Approximation Problem - Analysis and Simulation of a Joint-Implementation Program. Proceedings of the 3rd MATHMOD Vienna

12. M. Schroeder and S. Lingner (1999) Modelling Climate Change and its Economic Consequences - A Review. European Academy for the Study of Consequences of Scientific and Technological Advance, Graue Reihe Nr. 15

13. S.H. Tijs and T.S.H. Driessen (1986) Game theory and cost allocation methods. Management Science, 32: 1015-1028

14. S.H. Tijs (1987) An axiomation of the $\tau$-value. Mathematical Social Sciences, (13): 177-181 